This item was submitted to Loughborough's Research Repository by the author.

Items in Figshare are protected by copyright, with all rights reserved, unless otherwise indicated.

\title{
Using eye trackers as indicators of diagnostic markers: Implications from $\mathrm{HCl}$ devices
}

PLEASE CITE THE PUBLISHED VERSION

https://doi.org/10.1007/978-3-319-59424-8_29

\section{PUBLISHER}

C Springer International Publishing

\section{VERSION}

AM (Accepted Manuscript)

\section{PUBLISHER STATEMENT}

This work is made available according to the conditions of the Creative Commons Attribution-NonCommercialNoDerivatives 4.0 International (CC BY-NC-ND 4.0) licence. Full details of this licence are available at: https://creativecommons.org/licenses/by-nc-nd/4.0/

\section{LICENCE}

CC BY-NC-ND 4.0

\section{REPOSITORY RECORD}

Wilcockson, Thomas D.W.. 2019. "Using Eye Trackers as Indicators of Diagnostic Markers: Implications from HCl Devices”. figshare. https://hdl.handle.net/2134/37436. 


\title{
Using eye trackers as indicators of diagnostic markers: Implications from HCI devices.
}

\section{Thomas D W Wilcockson}

\begin{abstract}
Eye tracking allows psychologists to make broad distinctions between groups of participants. Therefore, it may be entirely possible to exploit these distinctions in order to create screening tools for potentially diagnosing various conditions. These diagnostic techniques may have a number of advantages over exiting techniques. However, in order to develop such screening tools it would be beneficial if eye tracking systems were easy to access and use. There are a number of ways to improve accessibility of eye trackers: affordability, transportation, ease of use,. This positional paper explores how HCI (Human Computer Interaction) eye trackers can be used for diagnostic purposes of psychological conditions.
\end{abstract}

Keywords Eye tracking - diagnosis - HCI - cognitive function - attentional bias

TDW Wilcockson

Lancaster University, Bailrigg, UK

e-mail: t.wilcockson@lancaster.ac.uk 


\section{Introduction}

Eye tracking allows psychologists to make broad distinctions between groups of participants (see Leigh \& Kennard, 2004). For example, eye movements can be indicative of whether a patient has had a concussion (Samandi, et al. 2015), has schizophrenia (Benson et al., 2012), or Alzheimer's disease (Crawford, et al., 2005). Therefore it may be entirely possible to exploit these distinctions in order to create screening tools for potentially diagnosing such conditions. There are a number of neural pathways involved in the generation of eye movements including the cerebrum, brainstem, and cerebellum. Therefore degeneration or damage in any of these areas would affect eye movements in a particular fashion which would be identifiable during eye tracking tasks and may indicate a specific disorder. Thus indicating the potential benefits which eye tracking could offer neurological and cognitive assessment.

At present eye tracking techniques have been used to compliment the assessment of patients (Anderson \& MacAskill, 2013). Eye movement can be measured in clinical settings without the use of eye tracking equipment. These observations by clinicians may involve simply asking a patient to follow the clinician's finger as it is moved from side to side. Such basic observations are not as accurate as using eye tracking technology but can give an indication of an amplification of a motor impairment of the disorder. Whereas laboratory-measured eye movements (using eye tracking technology) can provide rich and detailed identification of cognitive and/or neurological status and also monitor the progression of a disorder. Therefore there are advantages of using eye tracking equipment in the laboratory. This combined with the measurement of saccades enables the measurement of cognition as well as motor impairments associated with certain disorders. 
Eye movement research typically aims to measure two types of movements; vestibulo-ocular reflexes (VORs) or saccades. VORs are associated with the stabilising of the eye in relation to motion from the head. The second type of eye-movement is the saccade. This is a shift of the location of the eye in order to focus on areas of interest. It is this second type of eyemovement which is of particular interest due to its close relationship with attention, as attention may be affected by the cognitive impairments associated with, e.g., neurodegenerative disorders. As previously mentioned eye movement deficits can be observed clinically, however, it has generally been found that the recording of eye movements in a laboratory whilst performing an eye-tracking task is much more sensitive.

Traditionally a number of simple eye movement tasks have been used to observe cognitive function within patients. For example during a prosaccade task participants make a saccade (typically from a central location) toward a sudden onset target. Important measurements include the saccade latency, peak velocity, amplitude, and duration. During an anti-saccade task the participants fixate on a motionless target (such as a small dot) and a stimulus is then presented to one side of the target. The patient is asked to make a saccade toward the opposite direction of the stimulus. For example, if a stimulus is presented to the left of the motionless target, the patient should look toward the right. Failure to inhibit a reflexive saccade is considered an error. A reflex paradigm task involves directing the participant to look at a target as soon as it appears on the screen. By manipulating the onset time of the target a measure of inhibition can be obtained. Manipulating the gap can affect activity in the superior colliculus, which is associated with gaze fixation. This task allows the experimenter to change the demands of the task without changing the instructions the participant receives. A memory guided paradigm task requires more cognitive processing than the 
reflex paradigm. A target briefly appears on the screen, but the participant must suppress the reflexive saccade towards it until a second cue appears. Following the second cue the participant must fixate where the initial target was (which has subsequently vanished), so guided by memory alone.

These tasks have been successfully adopted to measure discrepancies between participant groups. For example Alzheimer's dementia (AD) typically require posthumous diagnosis in order to be confirmed. However, a diagnosis of probable $\mathrm{AD}$ is often given to patients with deficits in memory, one other cognitive domain, and everyday functioning. Utilising the antisaccade task with $\mathrm{AD}$ has demonstrated key distinctions between $\mathrm{AD}$ and non-dementia controls. Patients with AD typically make more uncorrected errors on the task in that they are much more likely to be unable to inhibit their attention toward the salient target (see Crawford et al., 2005). These findings would therefore suggest that the development of AD impairs executive control over eye movements (see Figure 1).
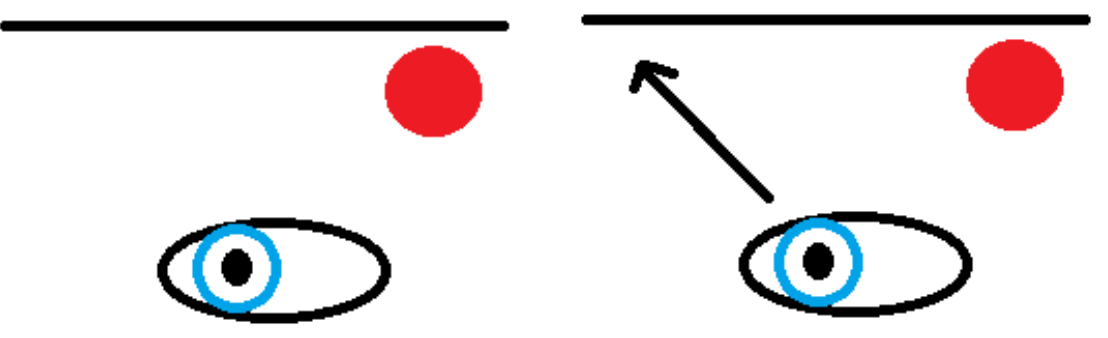

Fig. 1 A diagram of an antisaccade task trial. When a red circle appears on the screen the participant is told to look in the opposite direction of the stimulus.

By using a smooth pursuit task, where participants are told to follow a pattern on a computer screen, Benson et al (2012) found that 88 schizophrenia cases and 88 controls differed on the task in that the people with 
schizophrenia were impaired. It was also observed that the eye movement abnormalities appear to be stable traits, independent of current medication or mental states (see Figure 2).
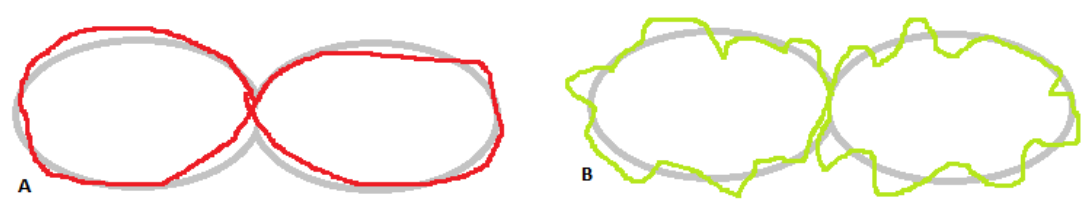

Fig. 2 The grey line indicates the path which the participant should follow with their eyes. Picture A demonstrates the performance of a control participant. Whereas picture B demonstrates an examples of the type of eye movement a Schizophrenic might display.

Samandani et al (2014) developed a technique for measuring concussion based upon the clinical examining of a patient who has sustained a head injury. Doctors traditionally would move a finger in front of the patient's eyes to notice any peculiarities in the movement of the eyes. During the eye movement task, participants would watch a video through a moving aperture, which enabled the measurement of ocular motility. It was found that concussion led to a decrease in performance (see Figure 3).
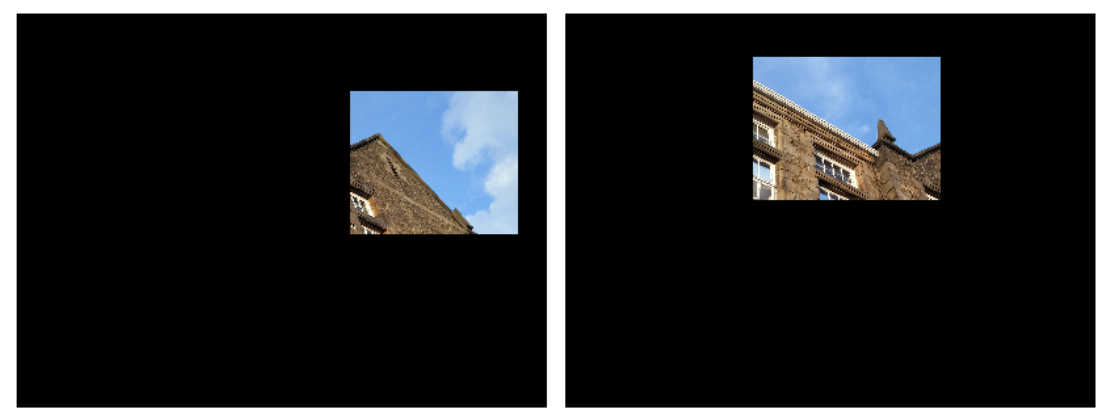
Fig. 3 A video is displayed on a screen. However, only a portion of the screen can be seen through an aperture.

Another use for eye movement techniques is the diagnosis of substance abuse disorders by measuring attentional biases. Attentional biases are the preferential processing of stimuli within the environment such that, e.g., a heavy alcohol drinker would direct their attention toward an alcoholrelated stimulus. Wilcockson \& Pothos (2015) observed that, using an eye movement gaze contingent paradigm ${ }^{1}$ whereby participants are told to avoid looking at stimuli, once an attentional bias has been developed for a substance, then processing of related stimuli is prioritised but also compulsory to attend to. Substance abuse can also distort perception of an environment so that there is a preoccupation with substance-related stimuli (Wilcockson $\&$ Pothos, 2016; see Figure 4). These findings all indicate a robustness of attentional bias: Substance abuse is facilitated by attentional biases which cause a preoccupation with substances, are hard to inhibit, and occur irrespective of context. These attentional biases have also been found to indicate whether substance abuse treatment has been successful or not as they indicate the likelihood of relapse (see Cox, Hoogan, Kristian, \& Race, 2002). Therefore, the ability to easily measure eye movement toward substancerelated stimuli could be useful for predicting treatment success rate.

All these findings taken together indicate that the vast array of eye movement techniques can be used to measure distinct clinical characteristics between participant/patient groups.

\footnotetext{
${ }^{1}$ A gaze contingency paradigm is a technique which allows a computer screen display to be modified dependent on where the viewer is looking
} 

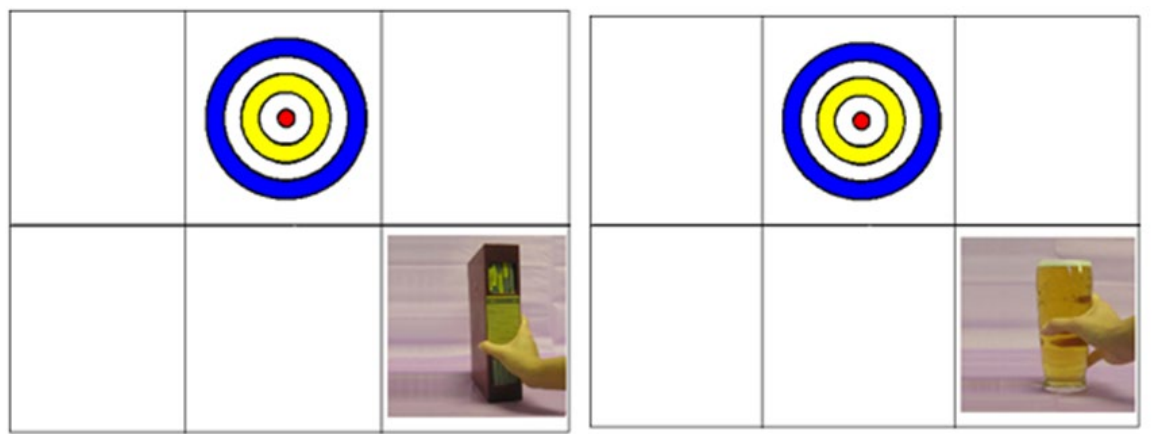

Fig. 4 Example of fixation region and distractor stimuli. The first image is an example of control distractor stimuli depicts a hand reaching for a folder (no grid lines were present in the experiment, they are shown here to represent the 6 sections of the screen which contain the fixation region and distractor stimuli). The second image is an example of a matched distractor stimuli. This example of alcohol distractor stimuli depicts a hand reaching for a pint. The matched control and experimental distractor stimuli are subtracted in order to make a 'difference' score. It is this score that is used to create the measures of attentional biases.

The importance of using laboratory tasks seems clear. These provide a number of advantages over clinical measure of eye movements. However, it is not also possible to utilise laboratory task on patients. As often patients are seen in clinical settings with relatively little space for equipment, or the time and skills required to set-up eye tracking equipment is lacking. Cost is also a big issue as eye tracking equipment can cost up to $\$ 35,000$ (compare to $\$ 99$ for an EyeTribe). Therefore, in order to realise the advantages that laboratory eye movement measures could provide a more practical system would apparently be necessary.

A number of companies produce eye tracking devices for the purposes of human computer interaction (HCI). These eye trackers are gener- 
ally used as a substitute for using a mouse or for controlling aspects of computer games. These devices are not as powerful as the eye trackers which psychologists would use for data collection. However, some psychological experiments do not require the accuracy and precision provided by expensive eye trackers.

Previous research has indicated that it is indeed possible to use the EyeTribe (Dalmaijer, 2014) and Tobii EyeX Controller (Gibaldi, et al., 2016) for data collection. These authors considered a number of aspects of the performance of these devices including accuracy, precision, sampling rate, fixation analysis, pupilometry, and saccade metrics. In each respect the HCI eye trackers performed admirably (however, saccade metrics seem to be the weakest aspects of these devices). In each regard the traditional data collection devices out-performed the HCI rivals. However, the HCI tools have the advantage of being cheap, easy to use, easy to transport, and are compact (see Figure 5).
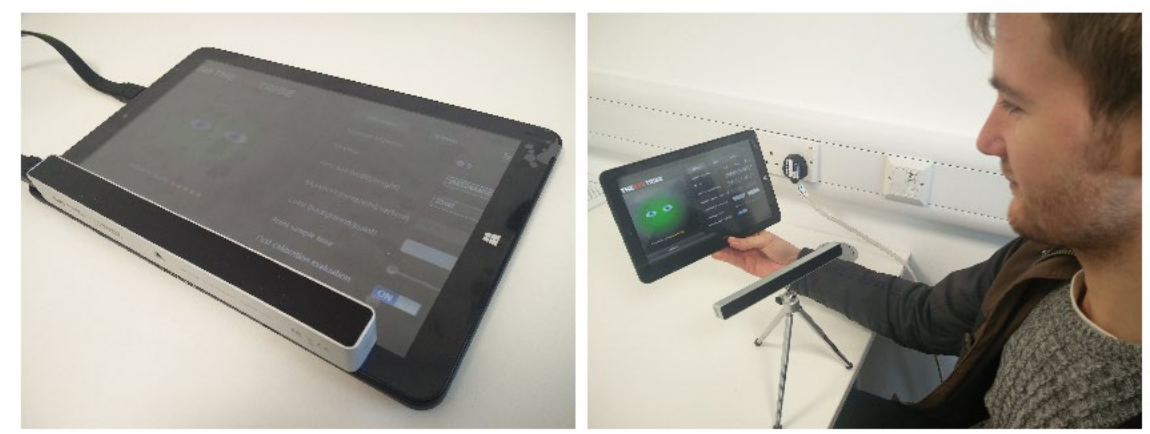

Fig. 5 A demonstration of the compact nature of the portable eye movement lab system. 
Therefore, it has already been demonstrated that this technology would be useful for data collection. The next step is to develop a straightforward and simple way of programming basic tasks which would be useful for the diagnosis of different participant groups in clinical settings. Of importance is developing the appropriate tasks which yield clinical diagnostic markers. But these tasks also need to be simple for clinicians whom are not experts in eye movement data collection and analysis.

The goal of this positional paper was to highlight the utility of $\mathrm{HCI}$ eye trackers in diagnosis and demonstrate a software that is very easy to use for this purpose. It was necessary to be able to easily change the stimuli and any demographic questions. The results also need to be instantly available on the screen following the procedure without the need of complicated analyses. This would enable the software and the eye tracker to be used in different situations and address all needs.

\section{Method}

An EyeTribe device was chosen to develop and explore this viability. The EyeTribe can be used with Windows or Macintosh computers. A Macintosh was used for the programming but a Windows 10 tablet to pilot the tasks. MatLab was used to control the stimulus events. The MatLab task was programmed in such a way that the stimuli could easily be changed by adding pictures to a corresponding desktop folder. This would enable a clinician to easily change the nature of the task e.g. by substituting alcohol-related stimuli for phobia-related stimuli in order to look at a patient with phobias rather than substance abuse. The task also contained demographic questions about the participant. These questions could be easily modified in the MatLab 
code. The task displayed basic graphical representations of the results on the screen following completion of the task.

Three different eye movement tasks were developed in order to measure different aspects of eye movement task design. The tasks were: a dot probe, an antisaccade, and a gaze contingent task. These tasks were chosen as they broadly represent the different eye movement tasks that have previously been used to demonstrate broad diagnostic distinctions between various patient-types. An example of one of the tasks can be seen in Figure 6.

How much alcohol

do you drink per

week?
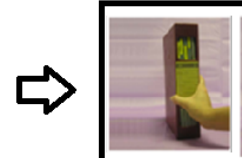

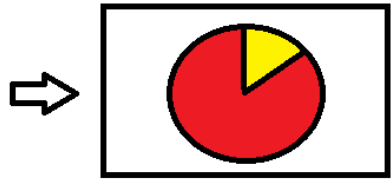

Fig. 6 This diagram of an example of a dot probe task. In this example alcohol usage is considered. Participants are first asked a question about their alcohol usage. The participant would respond using either a keyboard or touch screen. The eye movement task would then begin. In our task 10 trials were used. Each trial contained an alcohol and control stimulus. A number of eye movement metrics can be measured. It would be hypothesised that heavy alcohol drinkers would fixate on the alcohol stimulus before the control stimulus. This result can then be shown on the screen following the task. Here a pie chart shows that the participant fixated on the alcohol stimuli more than the control stimuli over the 10 trials.

\section{Results}

It was found that each of the tasks functioned appropriately with the EyeTribe device. The tasks could easily be modified for different circumstances 
and the results were clearly available on the screen. No technical ability was needed to set-up the equipment or to perform any analysis.

Specifically, a number of eye movement metrics are measurable with this system. Using the device it is possible to measure coordinates of eye movements on the screen together with timestamps. These can be used to infer a number of variables including fixations, dwell times, and saccades. The software can produce a number of different types of outputs including pie charts, bar graphs, and basic mean comparison statistics. The task could also be modified for different circumstances by changing small parameters related to the MatLab code. For example, changing the pictures in the relevant folders and changing the demographic questions. Therefore computer competency is minimal in order to utilise this system

\section{Conclusions}

In conclusion, it would appear that $\mathrm{HCI}$ eye trackers could contribute greatly to psychological and medical research. They provide a number of practical and procedural advantages over traditional psychological data collection devices. Of course HCI eye trackers are not able to fully replace the other systems. However, if rich data is not necessarily required then psychologists should consider using HCI devices. Using such systems could potentially enable a clinician to automatically recommend diagnoses for patients with certain conditions.

Following the demonstration that the method can be used simply and efficiently, the next step is to start using this methodology in clinical settings. Such a demonstration would have implications for future diagnosis of patients. Should the methodology be successful in clinical settings then 
the recommendation would be that clinicians start considering using such devices as part of diagnosing patients.

It should also be acknowledged that web-camera based eye tracking systems may also be a suitable alternative to HCI devices. Such systems may be even cheaper than HCI devices as web-cameras are readily available. Therefore web-cameras have the advantage of collecting data from many devices anywhere in the world with the possibility of downloading clinical tools from app stores. Xu et al. (2015) demonstrated the utility for such devices when fixation measurement is required. However, further development is required before saccades can be measured. This would be integral for some clinical measurements. However, web-cameras have been used in some clinical populations. Chau and Betke (2005) used web-cameras to enable severely paralysed patients to communicate with the computers. Therefore web-camera and HCI device eye tracking systems should both be considered for use by clinicians.

It is a limitation of this paper that there is no verification of our findings. However, the purpose of this paper is proof of concept and is intended to act as a positional paper. Here the theory and a potential methodology are presented. In future research it is hoped that the importance of this method can be demonstrated by conducting diagnostic assessments based on this.

In conclusion, eye movements can provide diagnostic markers for different participant groups. Exploiting these diagnostic markers would enable effective screening tools to be created. A hurdle in the way of deploying such eye movement tasks are the problems inherent with traditional eye tracking devices (e.g. cost, ease of use, time to set up, transportation). How- 
ever, here it is presented that it is possible to use HCI devices for the measurement of these diagnostic markers. Therefore, it is suggested that with further development clinicians should start to reconsider using eye movement as part of diagnosing in clinical settings.

Acknowledgements Thank you to Barrie Usherwood for his help in programming the eye movement tasks using the HCI equipment and Colleen McElhatton for her help with an earlier draft.

\section{References}

1. Anderson, T. J., \& MacAskill, M. R. (2013). Eye movements in patients with neurodegenerative disorders. Nature Reviews Neurology, 9(2), 74-85.

2. Benson, P. J., Beedie, S. A., Shephard, E., Giegling, I., Rujescu, D., \& Clair, D. S. (2012). Simple viewing tests can detect eye movement abnormalities that distinguish schizophrenia cases from controls with exceptional accuracy. Biological psychiatry, 72(9), 716-724.

3. Chau, M., \& Betke, M. (2005). Real time eye tracking and blink detection with usb cameras. Boston University Computer Science Department.

4. Cox, W. M., Hogan, L. M., Kristian, M. R., \& Race, J. H. (2002). Alcohol attentional bias as a predictor of alcohol abusers' treatment outcome. Drug and alcohol dependence, 68(3), 237-243

5. Crawford, T. J., Higham, S., Renvoize, T., Patel, J., Dale, M., Suriya, A., \& Tetley, S. (2005). Inhibitory control of saccadic eye movements and cognitive impairment in Alzheimer's disease. Biological psychiatry, 57(9), 1052-1060 
6. Dalmaijer, E. (2014). Is the low-cost EyeTribe eye tracker any good for research? (No. e585v1). PeerJ PrePrints

7. Gibaldi, A., Vanegas, M., Bex, P. J., \& Maiello, G. (2016). Evaluation of the Tobii EyeX Eye tracking controller and Matlab toolkit for research. Behavior research methods, 1-24.

8. Leigh, R. J., \& Kennard, C. (2004). Using saccades as a research tool in the clinical neurosciences. Brain, 127(3), 460-477.

9. Samadani, U., Farooq, S., Ritlop, R., Warren, F., Reyes, M., Lamm, E., ... \& Schneider, J. (2015). Detection of third and sixth cranial nerve palsies with a novel method for eye tracking while watching a short film clip. Journal of neurosurgery, 122(3), 707-720.

10. Wilcockson, T. D. W., \& Pothos, E. M. (2015). Measuring inhibitory processes for alcohol-related attentional biases: Introducing a novel attentional bias measure. Addictive behaviors, 44, 88-93.

11. Wilcockson, T. D., \& Pothos, E. M. (2016). How cognitive biases can distort environmental statistics: introducing the rough estimation task. Behavioural pharmacology, 27 (2 and 3-Special Issue), 165-172.

12. Xu, P., Ehinger, K. A., Zhang, Y., Finkelstein, A., Kulkarni, S. R., \& Xiao, J. (2015). Turkergaze: Crowdsourcing saliency with webcam based eye tracking. arXiv preprint arXiv:1504.06755. 